\title{
A novel tissue treatment to reduce mineralization of bovine pericardial heart valves
}

\author{
Bart Meuris, MD, PhD, ${ }^{\mathrm{a}}$ Herbert De Praetere, MD, ${ }^{\mathrm{a}}$ Marina Strasly, PhD, ${ }^{\mathrm{b}}$ Piero Trabucco, $\mathrm{PhD},{ }^{\mathrm{b}}$
}

John C. Lai, MS, ${ }^{b}$ Peter Verbrugghe, MD, ${ }^{a}$ and Paul Herijgers, MD, $\mathrm{PhD}^{\mathrm{a}}$

\section{ABSTRACT}

Objective: With the increasing use of bioprostheses worldwide, continuous efforts have been made to improve tissue durability. We introduce a new treatment for bovine pericardium combining octanediol-ethanol based phospholipid removal with taurine-based glutaraldehyde neutralization and storage in an aldehyde-free solution (FREE).

Methods: Treated tissues were evaluated by mechanical and biochemical characterization, phospholipid content, aldehyde levels, cell cultures on pericardial samples (L929 fibroblasts and human umbilical vein endothelial cells), rat subcutaneous implantations, and long-term juvenile sheep mitral valve implantations $(\mathrm{n}=3)$. Comparisons were made to glutaraldehyde-fixed bovine pericardium or to samples from commercially available biological valves (ie, Trifecta [St Jude Medical, Saint Paul, Minn] and Perimount Magna Ease [Edwards Lifesciences, Irvine, Calif]).

Results: FREE-treated pericardium had similar mechanical strength and biochemical properties as commercially available valves. Compared with glutaraldehyde-only samples, FREE-treated samples showed lower phospholipid levels $(P<.01)$, significantly better growth of L929 fibroblasts, and lower calcification levels in rat subcutaneous implants $(P<.01)$. Compared with samples from Linx- (Trifecta) and ThermaFix-treated (Perimount Magna Ease) valves, similar low levels of phospholipids were observed as were similar low calcification levels in subcutaneous implants, but tissue extractions from FREE-treated samples showed the lowest levels of extracted aldehydes $(P<.01)$. Mitral implants of FREE-treated valves in juvenile sheep had excellent hemodynamic behavior without any sign of degeneration or calcification at 5 months.

Conclusions: The new FREE treatment combines an adequate phospholipid reduction and aldehyde neutralization with storage in an aldehyde-free solution. This combination enhances the anticalcification properties and may thereby improve long-term durability of the tissue. (J Thorac Cardiovasc Surg

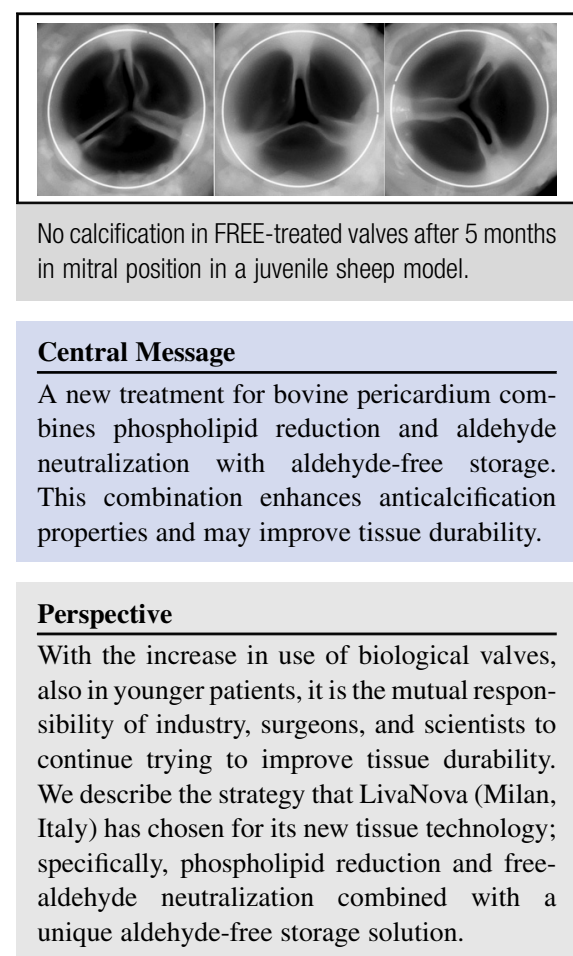

See Editorial Commentary page 207.

See Editorial page 196.
The use of biological heart valves is growing in many regions of the world. According to the Society of Thoracic Surgeons North American database, the proportion of isolated aortic valve replacements using biological prostheses

From the ${ }^{a}$ Department of Cardiovascular Sciences, Cardiac Surgery, KU Leuven, Leuven, Belgium; and bivaNova, Milan, Italy.

Received for publication June 3, 2017; revisions received Jan 15, 2018; accepted for publication Jan 29, 2018; available ahead of print March 20, 2018.

Address for reprints: Bart Meuris, MD, PhD, Department of Cardiovascular Sciences, Cardiac Surgery, University Hospitals Leuven, Herestraat 49, 3000 Leuven, Belgium (E-mail: bart.meuris@uzleuven.be).

$0022-5223 / \$ 36.00$

Copyright (C) 2018 by The American Association for Thoracic Surgery

https://doi.org/10.1016/j.jtcvs.2018.01.099 increased from $43 \%$ in 1997 to $78 \%$ in 2006 , whereas the proportion of mechanical valves implanted decreased from $50 \%$ to $20 \% .{ }^{1}$ When valve choice is discussed during patient consultation, younger and active patients prefer the benefits of a tissue valve. In addition, the current increase in

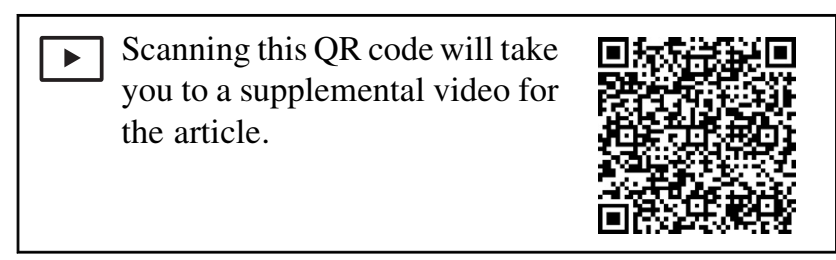




\section{Abbreviations and Acronyms \\ FREE = treatment for bovine pericardium that combines phospholipid reduction and aldehyde neutralization with aldehyde- free storage \\ $\mathrm{GA}=$ glutaraldehyde \\ HUVEC $=$ human umbilical vein endothelial cells ICP-OES $=$ induced coupled plasma-optical emission spectroscopy}

overall patients' life expectancy makes longer durability of tissue valves a necessity.

Tissue valves are primarily treated with a glutaraldehyde (GA) solution. However, GA has been shown to induce several negative effects, mainly through the persistence of unbound residual aldehyde groups ${ }^{2,3}$ that have the potential for direct binding to calcium, inducing lysis of cells surrounding the xenograft and resulting in the release of cellular debris and phospholipid cell membrane fragments ${ }^{4,5}$ and inhibiting endothelialization. ${ }^{6}$ Neutralization of these toxic aldehyde groups has been shown to reduce tissue mineralization. ${ }^{7}$ Cells within the xenograft are devitalized during glutaraldehyde fixation and subsequently lose their metabolic and active calcium efflux activity, resulting in passive influx of calcium into the cells. ${ }^{8}$ The calcium reacts with phosphorus found within remnants of the cellular debris, such as phospholipid membrane fragments, resulting in the formation of calcium phosphate apatite crystals within the devitalized cells. ${ }^{4}$ Removing or minimizing the presence of the phospholipids has been shown to reduce calcification of glutaraldehyde-fixed tissues. ${ }^{9-12}$

Antimineralization strategies for tissue valves available now on the market are addressing both phospholipid presence and/or unbound glutaraldehyde residuals in the fixed tissues. ${ }^{4}$ However, most tissue valves are typically still stored in aldehyde-containing solutions, thereby exposing the tissue to further aldehyde residuals that may persist despite the recommended rinsing processes. Here we describe a novel tissue process that utilizes an alcohol mixture for phospholipid removal combined with a poststerilization amino acid treatment for the neutralization of unbound aldehydes, and final packaging in an aldehyde-free storage solution (FREE treatment) (Video 1). Because the device is packaged in a solution free of aldehydes, an intraoperative rinsing procedure is not required and no residual toxicity is present. To assess the overall influence and efficacy of the FREE treatment, its performance was studied both in vitro and in vivo.

\section{METHODS}

\section{Basic Mechanistic Elements in FREE Treatment}

The 3 main steps of the FREE treatment are the phospholipid reduction, the neutralization of the residual aldehydes from tissue fixation and sterilization, and the storage in an aldehyde-free solution, which is unique in current

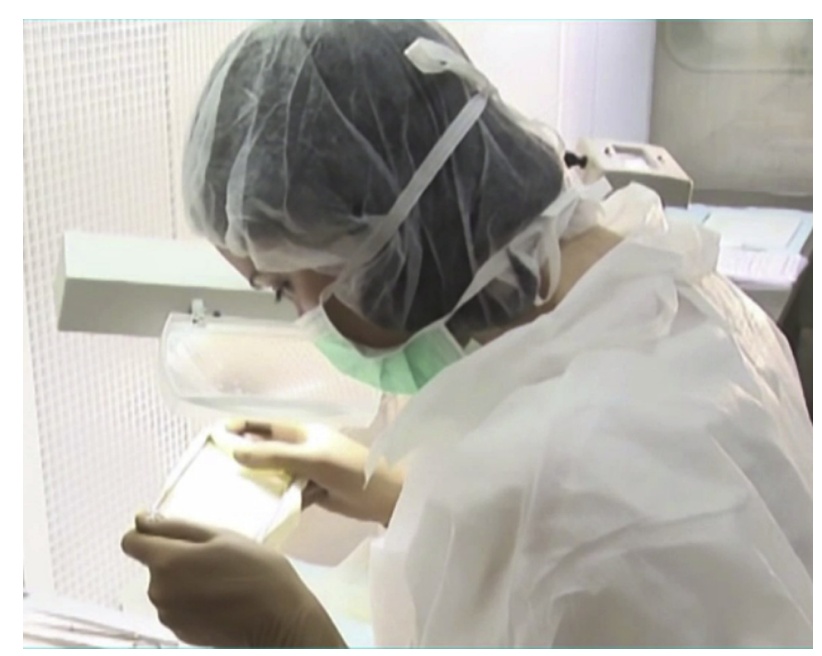

VIDEO 1. The animated video highlights the key elements of the new treatment: Efficient phospholipid removal, combined with aldehyde neutralization and aldehyde-free storage. A few basic mechanisms of valve tissue calcification are reviewed (adapted from Schoen FJ, Levy RJ. Calcification of tissue heart valve substitutes: progress toward understanding and prevention. Ann Thorac Surg. 2005;79:1072-80). Video available at: http://www.jtcvsonline.org/article/S0022-5223(18)30410-0/fulltext.

heart valve practice. Phospholipid reduction is obtained through a mix of short- and long-chain alcohols (ethanol and 1,2 octanediol), properly balanced to effectively dissolve the phospholipids of the cell membranes and cell debris present in the tissue. Effective aldehyde neutralization is then obtained at the very end of the valve manufacturing process by taurine, a small sulfonic acid with a highly reactive amino group able to bind free glutaraldehyde groups present in the tissue. The valve is stored in an aldehyde-free solution, a phosphate buffer with parabens, ready to be implanted. Process details are provided in US Patent No. 20140370599. ${ }^{13}$

\section{Pericardial Tissue Preparation}

Bovine pericardial sacs were procured from a commercial abattoir. Patches were treated with a GA solution at room temperature for 3 to 4 hours and then subjected to the FREE treatment.

\section{Mechanical Characterization by Ultimate Tensile Stress}

The tissue ultimate tensile stress testing was performed on dog boneshaped samples of pericardium. For each pericardial patch, 2 perpendicular samples were cut and subjected to tensile testing up to failure. The thickness of each specimen was measured to calculate the failure stress. A sample size of 24 pericardial patches was used (minimum sample size $=23$ based on power calculation). The test was performed in demineralized water at $37^{\circ} \mathrm{C}$ using a strain rate of $0.1 \mathrm{~mm} / \mathrm{sec}$ and a load cell of $250 \mathrm{~N}$. The failure stress was calculated (ultimate tensile strength $=\mathrm{F}_{\max } / \mathrm{A}_{0}$ ), where $\mathrm{A}_{0}$ is the sample cross-section and $\mathrm{F}_{\max }$ is the maximum force. The strain at failure $\varepsilon_{\mathrm{F}}$ was calculated as a percent $\left(\varepsilon_{\mathrm{F}}=100 \times \Delta 1 / 1_{0}\right)$, where $1_{0}$ is the length of the initial sample and $\Delta 1$ is the extension before failure. Samples of pericardial tissue from clinically available valves (LivaNova valves [Milan, Italy] with homocysteic acid-treated tissue) were used as control.

\section{Biochemical Characterization by Shrinkage Temperature Testing, Moisture, and Hydroxyproline Content}

On samples from 24 FREE-treated pericardial patches, shrink temperature testing, moisture content, and hydroxyproline content were evaluated. 
Pericardial tissue from current clinically available valves (LivaNova homocysteic acid-treated tissue $[\mathrm{n}=24]$ ) served as control. The shrinkage temperature corresponds to a sudden decrease of tissue length due to collagen fibers denaturation and is strictly related to the chemical properties of the biological tissue and independent from its morphologic and geometric characteristics (eg, thickness, collagen bundle orientation, and sampling area). For these reasons, the shrinkage temperature is the best parameter for the evaluation of the grade of tissue fixation. The degree of fixation of pericardium is related to an increase of the shrinkage temperature. The tissue moisture content was determined by measuring the loss in mass after sample dehydration at $100^{\circ} \mathrm{C}$. Hydroxyproline content was determined using a colorimetric reaction with 4-dimethylaminobenzaldehyde after sample hydrolysis with sulphuric acid. The hydroxyproline content (expressed as grams per $100 \mathrm{~g}$ ) was evaluated on dried samples. Hydroxyproline content on wet samples was also calculated from the result on dried samples by correcting for the tissue moisture content. The hydroxyproline content is indicative of the content of collagen fibers in the pericardial patch and therefore an indication of the presence of the main structural component in valvular tissue.

\section{Phospholipid and Unbound GA Content}

FREE-treated samples $(n=6)$ were compared with pericardial samples from either GA-fixed tissue without any further treatment (GA-only $[\mathrm{n}=18]$ ) or samples obtained from commercially available valves: Linxtreated tissue $(\mathrm{n}=3)$ from Trifecta valves (St Jude Medical, Saint Paul, Minn), ThermaFix-treated tissue $(\mathrm{n}=3)$ from CE-Perimount Magna Ease valves (Edwards Lifesciences, Irvine, Calif). Trifecta or Perimount valves were rinsed according to their respective instructions for use. After drying and mechanical pulverization of sample tissues, phospholipids were extracted using a chloroform methanol solution similar to that described by Stewart. ${ }^{14}$ Phospholipid content was measured using a spectrophotometric quantification after derivatization with ammonium ferrous thiocyanate. Unbound GA was qualitatively assessed by a fuchsine-based staining technique. For each of the 4 groups (GA-only, FREE, Linx, and ThermaFix), 3 samples were stained. Fuchsine stain turns dark blue in the presence of unbound aldehyde functional groups, whereas the lack of unreacted aldehyde groups will result in very little color change.

\section{Quantification of Extractable Aldehydes}

Valves (Trifecta and Perimount) were rinsed according to the product's instructions for use. FREE-treated valves were not rinsed before aldehyde extraction. The original storage solution of each valve was refrigerated $\left(2^{\circ} \mathrm{C}-8^{\circ} \mathrm{C}\right)$ and analyzed separately for aldehyde content. Each sample ( $\mathrm{n}=3$ for Trifecta and Perimount and $\mathrm{n}=6$ for FREE-treated tissue) was placed on a dry piece of lab paper, turned upside down to remove the excess of storage or rinsing solution, and then cut into 6 small pieces and placed into individual borosilicate glass containers with saline $(0.2 \mathrm{~g}$ wet tissue per milliliter saline). Samples were placed into an incubator at $37^{\circ} \mathrm{C} \pm 1^{\circ} \mathrm{C}$, shaken at 40 to $50 \mathrm{rpm}$ for 9 days in total. After 3,6 , and 9 days, respectively, samples were transferred to a new container and the saline extract was analyzed for aldehyde content. Determination of aldehyde content in the saline extracts and storage solution was done by high-performance liquid chromatography with diode array detector using Agilent Technologies Series 1100 High HPLC System (Santa Clara, Calif) after derivatization with 2,4-dinitrophenylhydrazine.

\section{In Vitro Cell Adhesion and Proliferation Assays}

Tissue samples subjected to the FREE treatment were briefly rinsed in Dulbecco's phosphate buffered saline before testing. GA-only treated tissue samples were stored in GA-based solutions and rinsed 3 times, 2 minutes each, in saline solution before testing. All samples were then placed in 12-well plates before seeding by cell lines. L929 fibroblast cells were seeded at $1.85 \times 10^{5}$ cells $/ \mathrm{mL}$ in $2.5 \mathrm{~mL}$ minimum essential media per well supplemented with $10 \%$ fetal bovine serum. They were grown for 72 hours at $37^{\circ} \mathrm{C}, 5 \%$ carbon dioxide, and $98 \%$ relative moisture with no change in media during this time. Four samples per treatment (FREE-treated vs GA-only) were tested. As a positive control, L929 cells were seeded without pericardial patches in polystyrene plastic plates alone. At the end of the 72 hours, microscopic determination of cell adhesion and growth was conducted by evaluating the presence of a cell monolayer, cell death, giant multinucleate cells, or any other changes in cell morphology. Cell viability on pericardial samples was assessed by MTT assay (dimethylthiazolyl-diphenyltetrazolium bromide). Human umbilical vein endothelial cells (HUVEC) were seeded at $10^{5}$ cells $/ \mathrm{mL}$ in the endothelial cell growth medium BulletKit (Lonza, Basel, Switzerland). Pericardial samples were coated with fetal bovine serum for 8 hours at $37^{\circ} \mathrm{C}, 98 \%$ moisture, and $5 \%$ carbon dioxide before culturing with HUVEC. Four samples per each treatment (FREE-treated vs GA only) were tested. Culture medium was not changed during the 72 hours of cell growth and observations were conducted at 4,48 , and 72 hours postseeding onto the pericardial samples.

\section{Preclinical Animal Studies}

All animal experiments were conducted upon approval of the relevant ethical committee for animal studies. In juvenile (4 weeks old) Wistar rats, pericardial patches $(1 \times 1 \mathrm{~cm})$ were implanted subcutaneously at 4 dorsal sites per rat. Four groups consisting of GA-fixed bovine pericardium without further treatment (GA only [ $\mathrm{n}=12]$ ), GA-fixed bovine pericardium subjected to the FREE tissue treatment $(n=12)$, bovine pericardial leaflets obtained from Trifecta valves $(\mathrm{n}=12)$, and bovine pericardial leaflets obtained from Perimount valves $(n=12)$ were tested. At 8 weeks postimplantation, rats were killed and explanted test articles were assessed for mineralization by semiquantitative scoring, including macroscopic examination, tactile evaluation, and quantitative calcium content by induced coupled plasmaoptical emission spectroscopy (ICP-OES). In addition, 3 juvenile sheep (aged 6-10 months at surgery) were implanted with bovine pericardial valves manufactured using the FREE treatment (Pericarbon MORE; LivaNova, Milan, Italy) in the mitral position for 20 weeks as described previously. ${ }^{15}$ Compared with the rat model where implanted tissue patches are subjected to a static environment, valve implantations in the orthotopic position of a large animal simulates clinical use more reliably because it accounts for the dynamic environment and blood contact. Animals were periodically monitored postoperatively and assessed clinically by a certified veterinarian. Transthoracic echocardiography was conducted at 1 week, 5 weeks, and 20 weeks postimplantation using previously described methods. ${ }^{16}$ At death, a necropsy was performed and radiographs of the explanted device conducted. Heart and valve pathology were evaluated macroscopically and by histologic examination (hematoxylin and eosin and Von Kossa staining) by an independent pathologist. Half of each leaflet was analyzed by ICP-OES to quantify the calcium present within the explanted tissue.

\section{Statistical Analyses}

All values are expressed as mean \pm standard deviation. Statistical analyses were performed using Statistica software version 13.2 (StatSoft Inc, Tulsa, Okla). Between-group comparisons were made using standard $t$ tests. Between-group comparisons for phospholipid content, extractable aldehydes, and calcium content were done using Kruskal-Wallis and Mann-Whitney $U$ tests.

\section{RESULTS}

\section{Mechanical and Biochemical Characterization of FREE-Treated Pericardium}

Figure 1 illustrates the values obtained from the mechanical and biochemical tests. As a reference point, values are also shown from current commercially available valves 


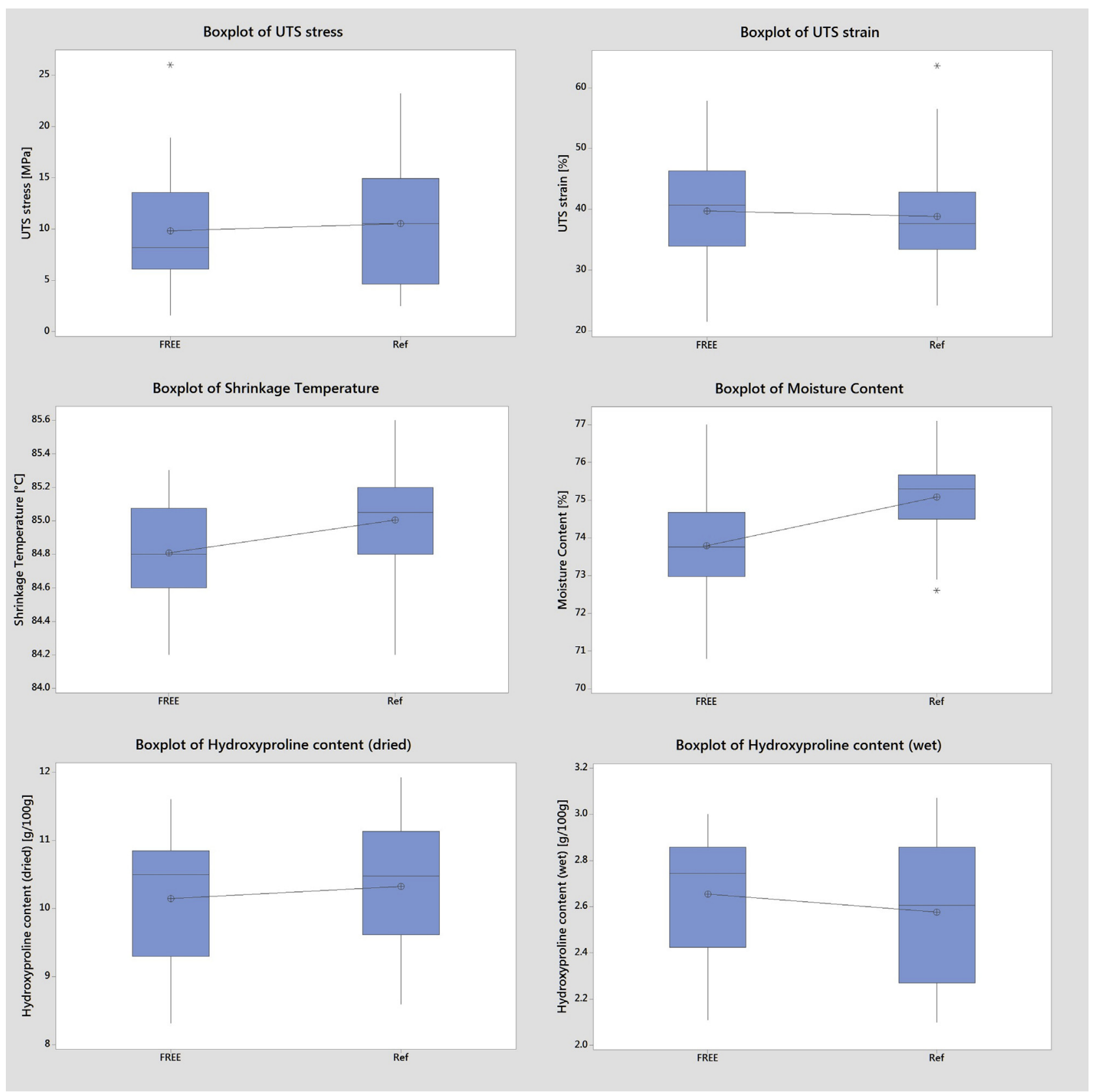

FIGURE 1. Boxplot charts of the tissue mechanical and biochemical data: Comparison between bovine pericardium treatment combining octanediolethanol based phospholipid removal with taurine-based glutaraldehyde neutralization and storage in an aldehyde-free solution $(F R E E)(\mathrm{n}=24)$ and reference samples $(\mathrm{n}=24)$. Average values are represented by circles. The blue boxes represent each data group through their quartiles. Lines extending vertically from the boxes (whiskers) indicate variability outside the upper and lower quartiles. UTS, Ultimate tensile stress. *Outliers.

(LivaNova valves). All of these values are in line with those obtained from pericardial patches from valves in clinical use.

\section{Determination of Phospholipid Content}

The phospholipid reduction process was effective at significantly reducing the phospholipid content in the pericardium. Figure 2 shows the phospholipid content for different pericardial samples: GA only $(n=18)$, FREE treated $(n=6)$, Linx treated $(n=3)$, and ThermaFix treated $(\mathrm{n}=3)$. The phospholipid content in FREE-treated tissue was significantly reduced by an average of $96 \%$ $(P<.001)$, which was consistent with the reduction of $94 \%$ reported by Pathak and colleagues,${ }^{10}$ using a similar alcohol-based treatment. No significant difference was present between FREE-treated samples and samples from Linx or ThermaFix treatments. 


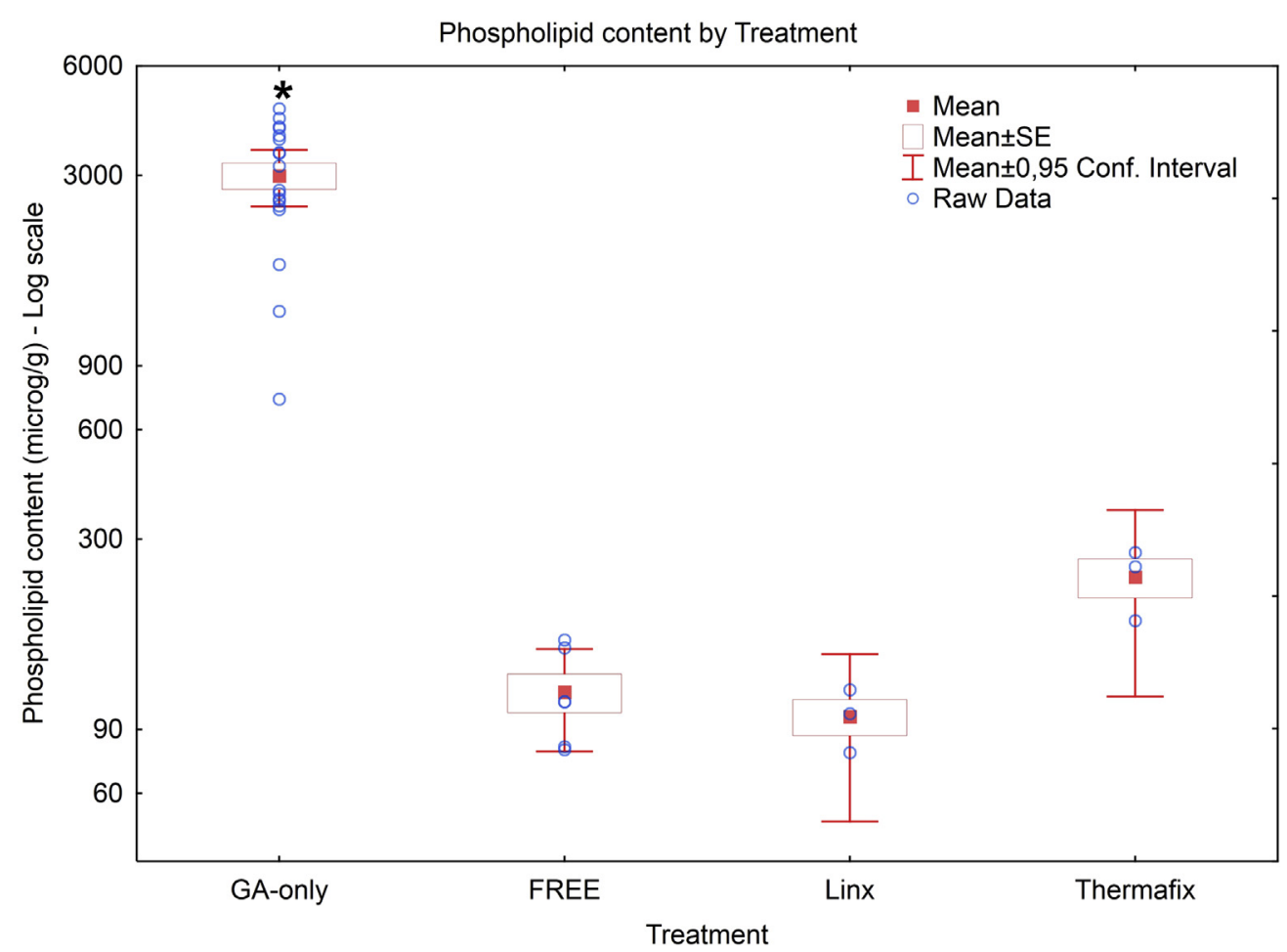

FIGURE 2. Phospholipid content for glutaraldehyde $(G A)$-only fixed tissue $(\mathrm{n}=18)$, tissue treated with a combination of octanediol-ethanol based phospholipid removal with taurine-based GA neutralization and storage in an aldehyde-free solution $(F R E E)(\mathrm{n}=6)$, Linx-treated tissue $(\mathrm{n}=3)$ from Trifecta valves (St Jude Medical, Saint Paul, Minn), and ThermaFix-treated tissue $(\mathrm{n}=3)$ from CE-Perimount Magna Ease valves (Edwards Lifesciences, Irvine, Calif). A significant difference was present between GA-only tissue and treated tissues, including the FREE-treated tissues ( ${ }^{*} P<.001$ vs all treated samples). No significant difference was present in phospholipid content between the treated tissues. SE, Standard error.

\section{Evaluation of Unbound Aldehydes: Colorimetric Assay}

All GA-only treated tissue samples demonstrated a positive response to fuchsine staining (dark violet color, positive control). Samples from Linx- and ThermaFix-treated valves, even when subjected to proper rinsing procedures, still had a positive response to fuchsine staining (Figure 3). All samples subjected to the novel FREE treatment showed very little fuchsine staining, with a final color similar to fresh pericardium, confirming very low unbound aldehyde content within these tissues.

\section{Evaluation of Extractable Aldehydes: Biochemical Assay}

Storage solutions. The quantification of aldehydes present in the valve storage solutions showed clear differences: the

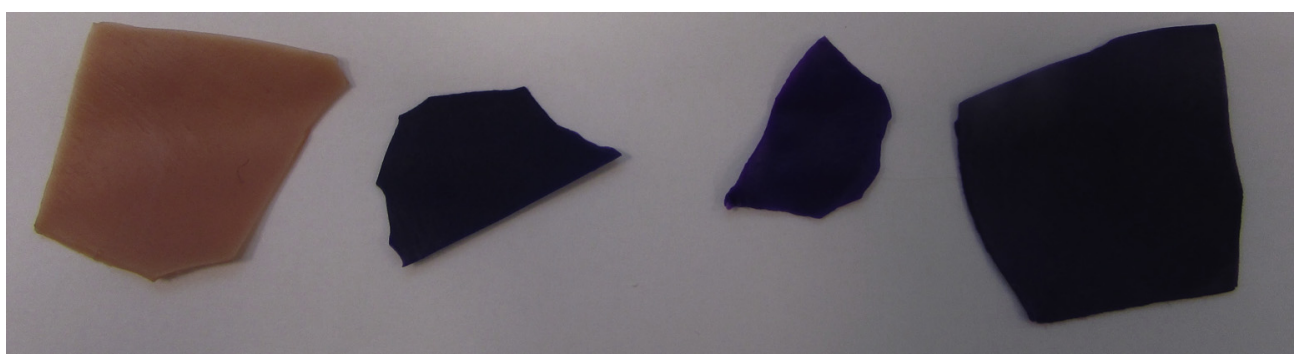

FIGURE 3. Sample from tissue treated with a combination of octanediol-ethanol based phospholipid removal with taurine-based glutaraldehyde neutralization and storage in an aldehyde-free solution (FREE) $(\mathrm{n}=3)($ far left $)$, ThermaFix $(\mathrm{n}=3)($ left $)$, Linx $(\mathrm{n}=3)($ right $)$, and glutaraldehyde only $(\mathrm{n}=3)(f a r$ right). The staining demonstrates the low level of unreacted free aldehyde functional groups after FREE treatment (far left sample) versus positive control samples from glutaraldehyde-only treated tissue (far right sample). Samples from Trifecta Linx-treated (St Jude Medical, Saint Paul, Minn) and CEPerimount Magna Ease ThermaFix-treated valves (Edwards Lifesciences, Irvine, Calif) (middle samples) clearly still stain positive for free aldehyde residuals despite proper rinsing. 


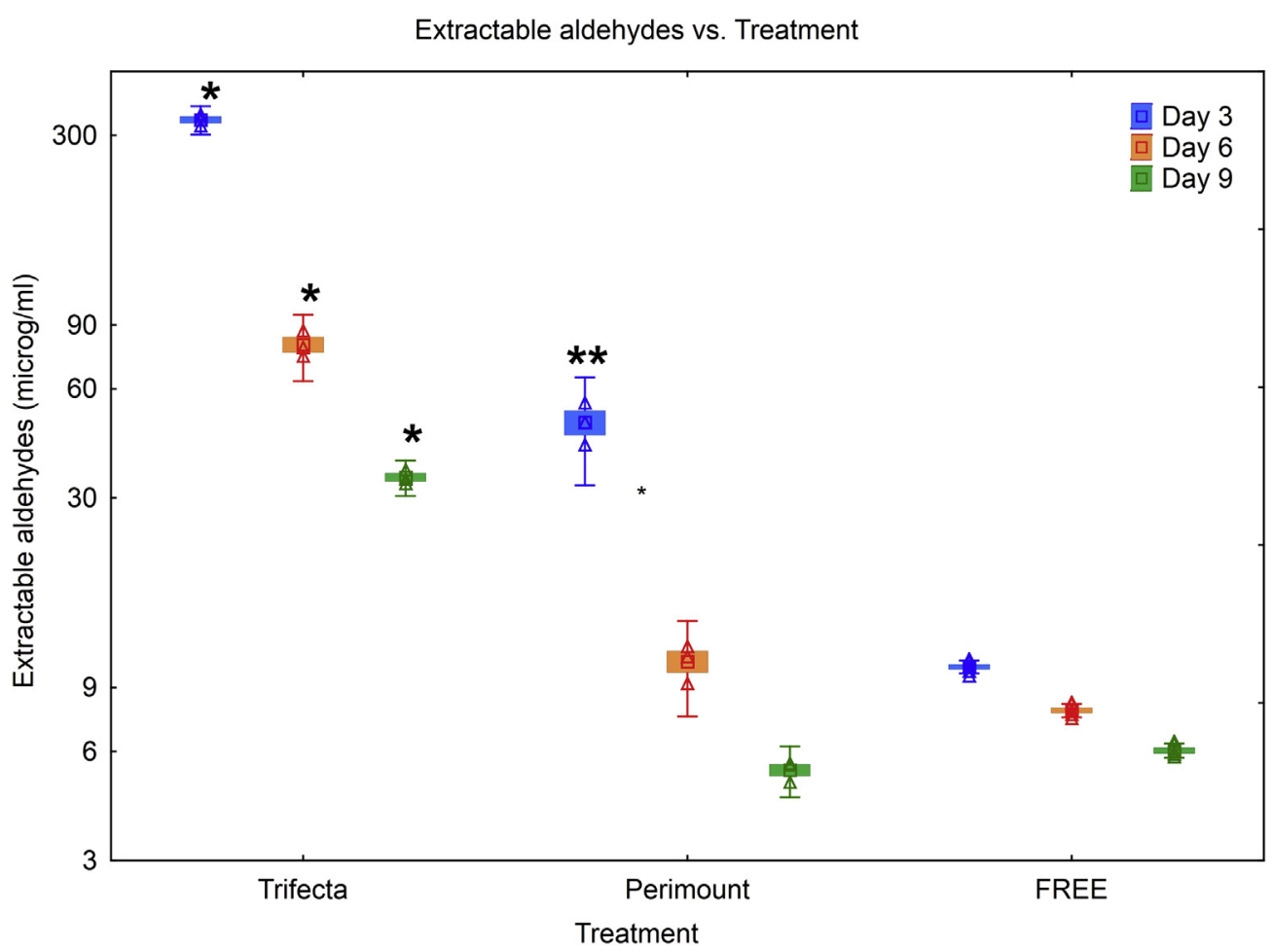

FIGURE 4. Aldehyde content in the extraction solution after day 3, day 6, and day 9. Compared with tissue treated with a combination of octanediolethanol based phospholipid removal with taurine-based glutaraldehyde neutralization and storage in an aldehyde-free solution $(F R E E)(\mathrm{n}=6)$, Trifecta valves (St Jude Medical, Saint Paul, Minn) $(\mathrm{n}=3)$ reveal significantly higher amounts of extractable aldehydes at all measured times $(* P<.01$ vs FREE-treated samples); CE-Perimount Magna Ease (Edwards Lifesciences, Irvine, Calif) valves $(\mathrm{n}=3)$ reveal a significantly higher aldehyde leak from the tissue only after day $3(* * P<.01$ vs FREE-treated samples).

storage solution for Trifecta and Perimount valves contained $6385 \pm 109 \mu \mathrm{g} / \mathrm{mL}$ and $4874 \pm 307 \mu \mathrm{g} / \mathrm{mL}$ aldehydes, respectively, explaining the requirement to thoroughly rinse these valves before implantation. The storage solution in FREE-treated valves contained only $1.2 \pm 0.05 \mu \mathrm{g} / \mathrm{mL}$ aldehydes $(P<.0001$ vs both Trifecta and Perimount valve storage solutions). The trace aldehyde content detected in the storage solution of FREE-treated valves is due to the small amount of free aldehydes diffusing from the GA-fixed pericardial tissue.

Aldehydes extractable from the tissue. Figure 4 illustrates the amount of aldehydes extracted from FREEtreated, Trifecta, and Perimount valves. The aldehydes extracted dropped over time in all samples. Compared with FREE-treated tissues, Trifecta valves had significantly higher amounts of extractable aldehydes at all measured intervals, whereas Perimount valves revealed a significantly higher aldehyde extraction after day 3 only.

\section{In Vitro Cell Adhesion and Proliferation Assays}

L929 fibroblasts seeded alone without pericardial patches and incubated in polystyrene plastic plates (positive control) showed absorbance values similar to those obtained from cell seeding on FREE-treated pericardial patches
( $P=$ not significant). The absorbance values observed after cell seeding on GA-only treated samples were significantly lower $(P<.001)$.

HUVEC cells were not able to adhere and grow on the pericardial samples in the same manner as L929 fibroblasts, and therefore it was not possible to conduct the same quantification analysis directly on pericardial samples as was done with the L929 cells. We did note that HUVEC cells were able to thrive directly adjacent to FREE-treated pericardium at 4, 48, and 72 hours after seeding, confirming the successful neutralization of GA in addition to a reduction in GA leaching adjacent to FREE-treated pericardium. In contrast, GA-treated pericardium showed a very toxic effect on the surrounding endothelial cells because all HUVEC cells were dead after 4 hours of incubation. At 48 hours postincubation, cells within wells containing FREE-treated samples were still observed to be viable but showed a decrease in proliferation when compared with control wells. The same behavior was observed at 72 hours.

\section{Rat Subcutaneous Study}

After 60 days of subcutaneous implantation there was no macroscopic sign of local adverse reaction observed at any 


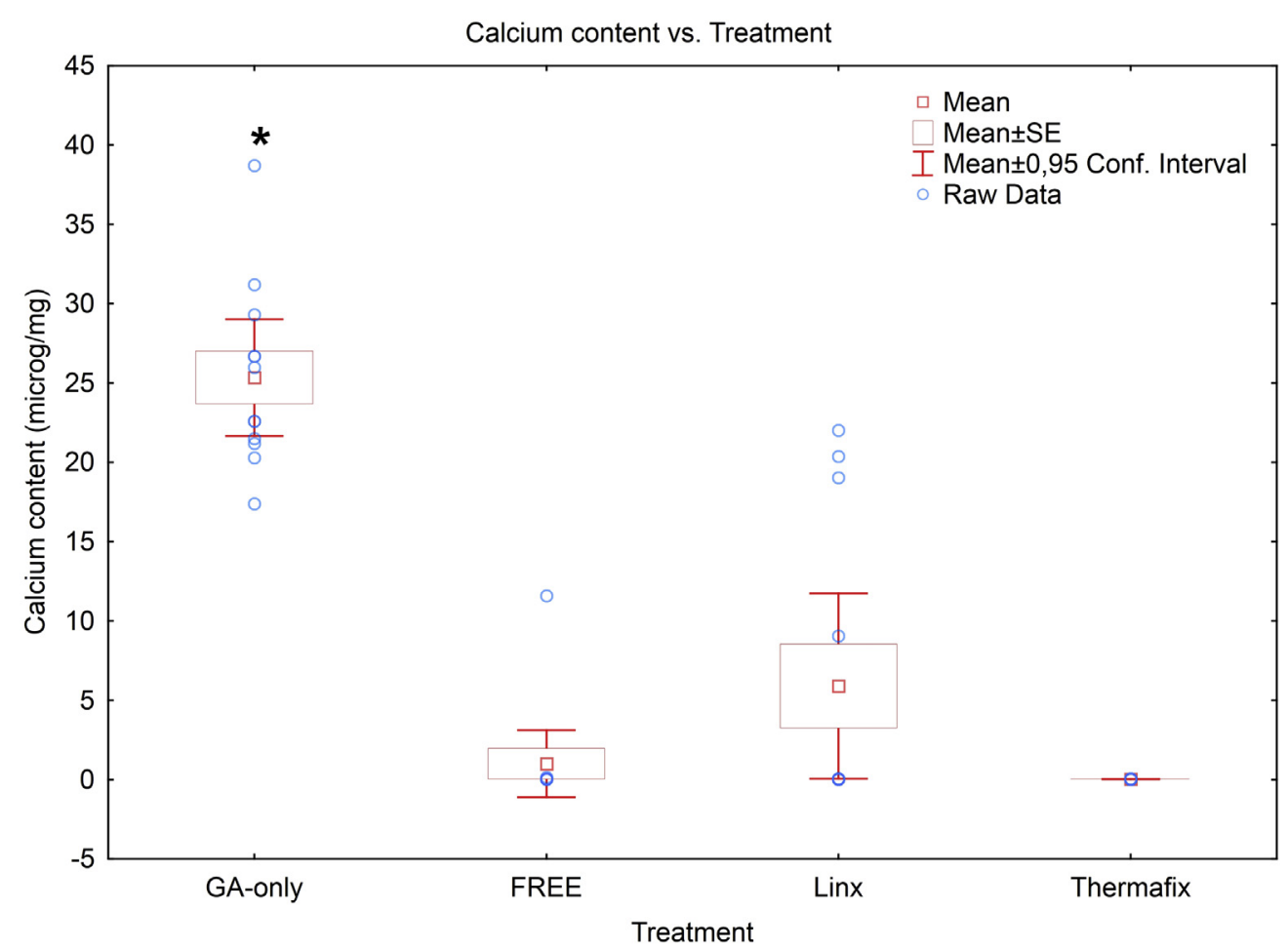

FIGURE 5. Calcium content determined by induced coupled plasma-optical emission spectroscopy within bovine pericardial tissues $(\mathrm{n}=12$ for each group) after 60 days subcutaneous implantation in juvenile rats. Untreated (glutaraldehyde [GA]-only) pericardium exhibited the highest levels of calcium content at $25.4 \mu \mathrm{g} / \mathrm{mg}$. All treated samples had significantly lower calcium content levels versus the GA-only samples $(* P<.001$ vs all treated samples). Between the treated samples, there were no significant differences. $S E$, Standard error; FREE, bovine pericardium treatment combining octanediol-ethanol based phospholipid removal with taurine-based glutaraldehyde neutralization and storage in an aldehyde-free solution.

site. Tactile evaluation of explanted tissue revealed that all FREE-treated samples preserved their original pliability and showed no indication of calcification, whereas the GA-only tissue had clearly stiffened. Similar to the FREE-treated pericardium, no samples excised from Perimount leaflets showed any macroscopic signs of mineralization, whereas 4 out of 12 tissue samples excised from Trifecta valves exhibited mild-to-moderate signs of mineralization (ie, whitish and stiffened areas extending over a major portion of the implanted sample). Consistent with the qualitative assessment, the quantitative assessment of calcium content within explanted tissues by ICP-OES revealed significant mineralization within GA-only fixed bovine pericardial samples, with a mean calcium concentration of $25.4 \pm 5.8 \mu \mathrm{g} / \mathrm{mg}$ (Figure 5). Extremely low calcium concentrations were observed in FREE-treated tissues $(0.05 \pm 0.03 \mu \mathrm{g} / \mathrm{mg} ; P<.001$ vs GA only). A similar low level of calcium was observed for tissues excised from Perimount valves $(0.04 \pm 0.01 \mu \mathrm{g} / \mathrm{mg})$, whereas the average calcium content measured within Trifecta samples were slightly higher $(5.9 \pm 9.2 \mu \mathrm{g} / \mathrm{mg})$.

\section{Juvenile Sheep Study}

Transthoracic echocardiography performed at 1,5 , and 20 weeks after valve implantation showed no major abnormalities or change over time, with all animals showing good recovery and satisfactory heart functions over the entire 20 -week period. From 1 to 20 weeks postoperatively, the mean gradient evolved from $3 \pm 1 \mathrm{~mm} \mathrm{Hg}$ to $5 \pm 3 \mathrm{~mm} \mathrm{Hg}$, and the peak gradient from $6 \pm 2 \mathrm{~mm} \mathrm{Hg}$ to $9 \pm 4 \mathrm{~mm} \mathrm{Hg}$. No valvular or paravalvular leakage was found. At explantation, satisfactory healing response to the implant was observed and FREE-treated valves had no major abnormalities on both the inflow and outflow sides with pliable leaflets and no visible signs of dystrophic calcification (Figure 6). Radiographic imaging of the explanted valves revealed practically no signs of dystrophic mineralization at 20 weeks after implantation (Figure 7). The mean calcium content of the leaflets was $4.5 \pm 1.8 \mu \mathrm{g} / \mathrm{mg}$ dry weight (range, 3.11-6.5 $\mu \mathrm{g} / \mathrm{mg}$ ).

\section{DISCUSSION}

The presence of unbound aldehyde residuals in biological tissue has several detrimental effects and can accelerate bioprosthetic tissue calcification significantly. ${ }^{2-7}$ Neutralization of aldehyde functional groups in GA-fixed valve tissue enhances tissue durability, lowers calcification potential, and even stimulates partial endothelialization with potential added benefits such as reduced thrombogenicity. ${ }^{17,18}$ Next to this, the presence of phospholipids inside the 


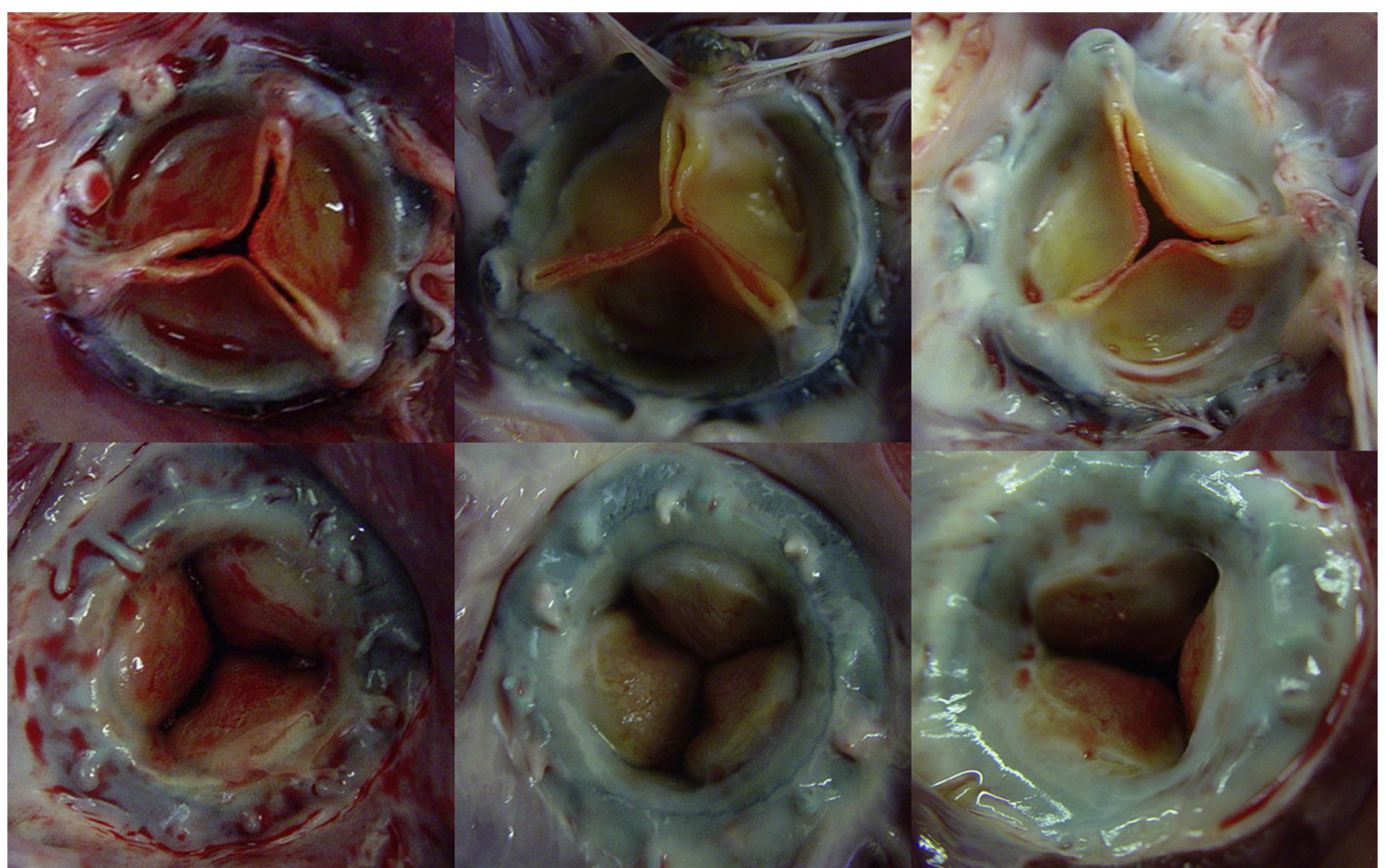

FIGURE 6. Outflow (upper panels) and inflow (lower panels) sides of mitral valve prostheses made of bovine pericardium subjected to the treatment combining octanediol-ethanol-based phospholipid removal with taurine-based glutaraldehyde neutralization and storage in an aldehyde-free solution treatment after 20 weeks of implantation in juvenile sheep (valve model is Pericarbon MORE [LivaNova, Milan, Italy]). Excellent healing response to the grafts was observed, with no signs of tissue thickening, tears, thrombosis, or macroscopic evidence of leaflet stiffening or mineralization.

tissue are well known potential early nuclei of calcification. $^{8-12}$ Several currently clinically available valves have aldehyde neutralizing strategies and/or lipid removal treatments. Unfortunately, after the treatment all current valves are stored in aldehyde-containing liquids.
This exposes the tissue again to the detrimental effects of remaining free aldehydes, even when preimplant rinsing procedures are performed following the instructions for use.

In this study, we report on test results from a novel tissue treatment that combines an octanediol-ethanol based
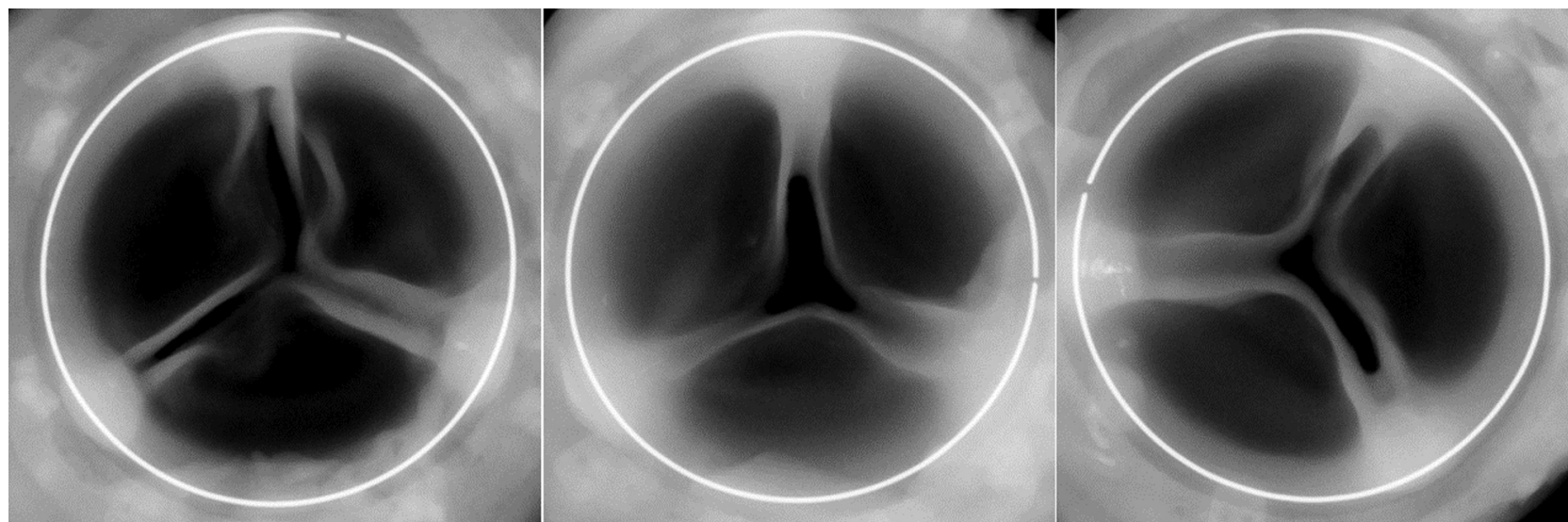

FIGURE 7. Radiographic images of bioprosthesis treated with a combination of octanediol-ethanol-based phospholipid removal with taurine-based glutaraldehyde neutralization and storage in an aldehyde-free solution (valve model is Pericarbon MORE [LivaNova, Milan, Italy]) after 20 weeks of implantation in a mitral position in juvenile sheep. 
phospholipid reduction and taurine-based glutaraldehyde neutralization with the use of an aldehyde-free storage solution.

The mechanical evaluation and biochemical analyses of the FREE-treated pericardium revealed similar results to those of valves that are already in clinical use. This illustrates the proper fixation and preparation of the tissue, making it suitable for further investigation and potential clinical applications. The FREE treatment has been shown to be effective in reducing the phospholipid content in pericardial tissue by up to $96 \%$, an extent of lipid removal similar to Linx- or ThermaFix-treated tissue.

On top of successful removal of phospholipids, fuchsine staining and aldehyde extraction assays demonstrated improvements in the removal and neutralization of unbound glutaraldehyde in FREE-treated tissues when compared with other clinical tissues. Substantial amounts of aldehydes were extracted from both Linx- and ThermaFixtreated valves, even when properly rinsed in saline. The combination of this new treatment with final storage in an aldehyde-free solution minimizes aldehyde exposure optimally and thereby eliminates a potential source of device toxicity. Routine GA-fixed tissue (that is also subsequently stored in GA), retains a high level of toxicity toward both fibroblast and HUVEC cells even if washed 3 times in saline solution. FREE-treated tissue shows no toxic effect with better in vitro cell adhesion and growth. It is not possible at this moment to state that this observation might lead to improved endothelialization of the whole implant.

The efficacy of the FREE treatment was also assessed through in vivo studies. The rat study has shown that FREE treatment effectively reduces tissue mineralization in comparison with untreated controls. Tissue samples from commercially available heart valve bioprostheses (Linx and ThermaFix treatments) have shown similar behavior in this small animal model. Further confirmation of the safety of the tissue was provided by the data gathered from mitral valve implantations in a large animal model. Despite the limited number of animals implanted, this preliminary study has shown that the FREE treatment applied to bovine pericardium does not have an effect on the functional performance of the valve while reducing the dystrophic mineralization typically observed after a few weeks of implantation in this model. ${ }^{15}$ Although no statistically significant conclusion can be obtained from the limited number of mitral implants, even in the highly challenging condition of 20 weeks' implantation in the mitral position of juvenile sheep, the FREE treatment appeared to be safe and durable without any major signs of mineralization or degeneration.

\section{Limitations}

The biomechanical tests of the FREE-treated tissue were compared with LivaNova tissue only; no comparisons to biomechanical properties of tissues from competitor valves were done. Only uniaxial tensile testing was performed; biaxial testing was not conducted. The sheep mitral implants served as a pilot study only. These first animals represent the proof-of-concept study performed before further in vivo implantations on a larger set of animals and including a control group.

\section{CONCLUSIONS}

The new FREE treatment resulted in bovine pericardial tissue with mechanical characteristics consistent with what is used in today's clinically used valves. This treatment strategy allowed cell adhesion and cell viability on tissue and mitigated mineralization propensity of GA-fixed tissues, as shown in both a small and large animal models. The reduction of phospholipids, together with the neutralization of free aldehydes, will beneficially influence the anticalcification properties and thereby enhance durability. The aldehyde-free storage solution is unique in current practice and avoids any new exposure of the implant to the toxicity of aldehyde solutions. Future studies will be conducted to further investigate the beneficial properties of the FREE treatment and to allow this treatment to be applied to commercially available valves.

\section{Conflict of Interest Statement}

This research project was sponsored by LivaNova, Milan, Italy, which provided all necessary funding for the study. Dr Meuris is a consultant to LivaNova. Drs Strasly, Trabucco, and Lai are LivaNova employees. All other authors have nothing to disclose with regard to commercial support.

\section{References}

1. Brown JM, O'Brien SM, Wu C, Sikora JAH, Griffith BP, Gammie JS. Isolated aortic valve replacement in North America comprising 108,687 patients in 10 years: changes in risks, valve types, and outcomes in the Society of Thoracic Surgeons national database. J Thorac Cardiovasc Surg. 2009;137:82-90.

2. Levy RJ. Glutaraldehyde and the calcification mechanism of bioprosthetic heart valves. J Heart Valve Dis. 1994;3:101-4

3. Gong G, Ling Z, Seifter E, Factor SM, Frater RW. Aldehyde tanning: the villain in bioprosthetic calcification. Eur J Cardiothorac Surg. 1991;5:288-99; discussion 293.

4. Thiene G, Valente M. Anticalcification strategies to increase bioprosthetic valve durability. J Heart Valve Dis. 2011;20:37-44.

5. Simionescu DT. Prevention of calcification in bioprosthetic heart valves: challenges and perspectives. Expert Opin Biol Ther. 2004;4:1971-85.

6. Hoffman D, Gong G, Liao K, Macaluso F, Nikolic SD, Frater RWM. Spontaneous host endothelial growth on bioprostheses. Influence of fixation. Circulation. 1992;86:75-9.

7. Valente M, Pettenazzo E, Thiene G, Molin GM, Martignago F, De Giorgi G, et al. Detoxified glutaraldehyde cross-linked pericardium: tissue preservation and mineralization mitigation in a subcutaneous rat model. J Heart Valve Dis. 1998;7:283-91.

8. Rao KP, Shanthi C. Reduction of calcification by various treatments in cardiac valves. J Biomater Appl. 1999;13:238-68.

9. Jorge-Herrero E, Fernandez P, de la Tone N, Escudero C, García-Páez JM, Buján J, et al. Inhibition of the calcification of porcine valve tissue by selective lipid removal. Biomaterials. 1994;15:815-20.

10. Pathak CP, Adams AK, Simpson T, Phillips RE, Moore MA. Treatment of bioprosthetic heart valve tissue with long chain alcohol solution to lower calcification potential. J Biomed Mater Res A. 2004;69:140-4. 
11. Pettenazzo E, Valente M, Thiene G. Octanediol treatment of glutaraldehyde fixed bovine pericardium: evidence of anticalcification efficacy in the subcutaneous rat model. Eur J Cardiothorac Surg. 2008;34:418-22.

12. Vyavahare N, Hirsch D, Lerner E, Baskin JZ, Schoen FJ, Bianco R, et al. Prevention of bioprosthetic heart valve calcification by ethanol preincubation: efficacy and mechanisms. Circulation. 1997:95:479-88.

13. Strasly M, Rolando G, inventors; Dideco SpA, assignee. Method for preparing biological tissues for use in biological prostheses. US Patent No. 20140370599. December 18, 2014.

14. Stewart JCM. Colorimetric determination of phospholipids with ammonium ferrothiocyanate. Anal Biochem. 1980;104:10-4.

15. Flameng W, Meuris B, Yperman J, De Visscher G, Herijgers P, Verbeken E. Factors influencing calcification of cardiac bioprostheses in adolescent sheep. $J$ Thorac Cardiovasc Surg. 2006;132:89-98.
16. De Vleeschauwer S, De Praetere H, Meuris B, Herijgers P, Herregods M-C. Postoperative echocardiographic evaluation of bioprosthetic mitral valve implantation in sheep. Lab Anim. 2015;49:20-9.

17. Leukauf C, Szeles C, Salaymeh L, Grimm M, Grabenwöger M, Losert U, et al. In vitro and in vivo endothelialization of glutaraldehyde treated bovine pericardium. J Heart Valve Dis. 1993;2:230-5.

18. Gulbins H, Pritisanac A, Pieper K, Goldemund A, Meiser BM, Reichart B, et al. Successful endothelialization of porcine glutaraldehydefixed aortic valves in a heterotopic sheep model. Ann Thorac Surg. 2006; $81: 1472-9$.

Key Words: heart valves, anticalcification treatment, durability 